\title{
MECHANISM OF ORIENTATION SELECTIVITY DURING GRAIN GROWTH OF SECONDARY RECRYSTALLIZATION IN Fe-3\%Si ALLOY
}

\author{
Y. USHIGAMI *, T. KUBOTA and N. TAKAHASHI \\ Electromagnetic Materials, Steel Research Laboratories, \\ Nippon Steel Corporation, 20-1 Shintomi, Futtsu, Chiba-ken, 293-8511 Japan
}

(Received in final form 28 September 1997)

Selective growth of $\{110\}\langle 001\rangle$ grains in the temperature gradient annealing has been studied in $\mathrm{Fe}-3 \% \mathrm{Si}$ alloy. As grains grow, the average deviation angle from the ideal $\{110\}\langle 001\rangle$ orientation becomes smaller and orientation distribution changes corresponding to that of coincidence grains in the matrix. Secondary recrystallization temperature depends on the orientation of secondary recrystallized grain and sharper $\{110\}\langle 001\rangle$ grains grow preferentially at lower temperatures.

These phenomena are explained by modified Hillert's model of grain growth. Interfacial energy of coincidence boundary is lower than that of general boundary. Therefore, sharper $\{110\}\langle 001\rangle$ grains, which have higher frequency of coincidence grains in the primary recrystallized matrix, suffer lower pinning effect from the second phase particles and thus grow preferentially at lower temperatures.

Keywords: Silicon steel; Secondary recrystallization; Texture; Inhibitor; Coincidence boundary; Grain growth

\section{INTRODUCTION}

Grain oriented silicon steel is a soft magnetic material widely used for transformer cores. The magnetic properties are closely related to the sharpness of $\{110\}\langle 001\rangle$ texture, i.e. Goss texture, which is evolved by secondary recrystallization. Iron crystals have an anisotropy in

\footnotetext{
* Corresponding author.
} 
magnetization and the axis of easy magnetization is $\langle 001\rangle$. If the preferred orientation of $\{110\}\langle 001\rangle$ is developed, the product has superior magnetic properties, such as low core loss, low magnetostriction and high permeability, along the rolling direction.

The principal process for developing Goss texture was invented by Goss in 1934 (Goss, 1934; 1935) and industrialized by ARMCO in the 1940s [CGO: conventional grain oriented silicon steel] (Littmann, 1967). The average deviation angle of $\langle 001\rangle$ axis is $7^{\circ}$ from the rolling direction. High permeability grain oriented silicon steels (average misorientations of $3-4^{\circ}$ ) were developed mainly by NSC in the 1960 s [HI-B] (Taguchi and Sakakura, 1969; Yamamoto et al., 1972). The major differences between these processes are in the following two respects: (1) primary recrystallization texture; the CGO process involves two stage cold rolling with an intermediate recrystallization annealing and the HI-B process a single stage cold rolling with heavy reduction of about $87 \%$ compared to the final reduction of about $50 \%$ in the CGO process. Depending on the difference of these final cold rolling reductions, $\{110\}\langle 001\rangle$ and $\{111\}\langle\mathrm{uvw}\rangle$ are the major components of the primary recrystallization texture in the CGO process. On the other hand, $\{110\}\langle 001\rangle$ is negligible and $\{111\}\langle 112\rangle$ is the major component in the HI-B process (Flowers and Heckler, 1976; Mishra et al., 1986; Matsuo, 1989). (2) inhibitor; CGO utilizes MnS and HI-B AIN in addition to $\mathrm{MnS}$ for the grain growth inhibitor of primary recrystallized matrix.

Many studies have been reported on secondary recrystallization in $\mathrm{Fe}-3 \% \mathrm{Si}$ alloy. Some researchers (Matsuo et al., 1982; Inokuti et al., 1982; Mishra et al., 1984) investigated the origin of Goss nuclei and claimed that the size advantage of the nuclei takes an important role in the evolution of Goss texture. Most of these researches were related to the CGO-type process. On the other hand, in the case of HI-B type process, others (Shinozaki et al., 1978; Harase et al., 1986; Nakayama and Ushigami, 1986; Rouag and Penelle, 1989) investigated the relationship between the secondary recrystallization texture and primary recrystallization texture and claimed that some, special boundaries (for example, $\Sigma 9$ coincidence boundary) play an important role in selective growth of Goss grains.

Present authors have studied the effect of cold rolling reduction on primary and secondary recrystallization texture evolution and have 
found that the frequency of coincidence boundaries increases with cold rolling reduction (Arai et al., 1996). This fact suggests that the former oriented nucleation mechanism dominates the preferred growth of Goss grains in the CGO type process and the latter oriented growth mechanism in the HI-B type process, which is practically more effective for texture control. In this consequence, the present authors have also found that the sharpest Goss texture (average misorientation of $1^{\circ}$ or so) evolves when grain growth of secondary recrystallization is accelerated by temperature gradient annealing in the HI-B process (Nozawa et al., 1986). However, the precise observation of grain growth behavior in the temperature gradient annealing has not been performed.

The objective of this paper is to investigate the grain growth behavior of secondary recrystallized Goss grains in the temperature gradient annealing and clarify the mechanism of orientation selectivity in the secondary recrystallization process.

\section{EXPERIMENTAL PROCEDURE}

The starting material was $\mathrm{Fe}-3 \% \mathrm{Si}$ primary recrystallized sheet of $0.2 \mathrm{~mm}$ thickness with the following chemical compositions; Si: 3.3, C: $0.002, \mathrm{Al}: 0.03, \mathrm{~N}: 0.007$ mass $\%$. The processing steps involved a hot rolling, an annealing, a single stage cold rolling with the reduction of $90 \%$ and a primary recrystallization and decarburization annealing. In order to inhibit the grain growth of primary recrystallized matrix effectively, nitriding treatment was performed to increase the nitrogen content to 0.05 mass\% (Ushigami et al., 1996). The sheet was then heated up to $1200^{\circ} \mathrm{C}$ in the temperature gradient of $0.5^{\circ} \mathrm{C} / \mathrm{mm}$ perpendicular to the rolling direction in $\mathrm{N}_{2}-\mathrm{H}_{2}$ mixture gas.

Orientations of the secondary recrystallized grains were measured by back-reflection Laue diffraction method. The complete $\{100\}$ pole figures were measured by X-ray measurement from the surface (1/5th of the thickness) and center layer of the specimen. Primary recrystallization texture was determined by three dimensional texture analysis by vector method (Ruer and Baro, 1977) and then orientation relationships between the potential secondary recrystallized grains and primary recrystallization texture were analyzed. 


\section{RESULTS AND DISCUSSIONS}

\subsection{Texture Evolution During Grain Growth}

Figure 1 shows the macrostructure of the secondary recrystallized grains annealed in the temperature gradient. It can be seen that several secondary recrystallized grains nucleated in the higher temperature region and grew along the temperature gradient direction. It has been confirmed that the grains grow along the temperature gradient direction regardless of the cold rolling direction. As grains grew, some of the grains grew preferentially to form the columnar structure. Orientation of individual secondary grains was measured and represented as the deviation angles from the ideal Goss orientation around three axes; normal direction of the sheet (ND), rolling direction (RD), and transverse direction (TD). During the grain growth, the average deviation angle from the ideal Goss orientation became smaller and orientation distribution (around three axes of ND, RD, TD) changed; the initial orientation distribution was qualitatively estimated as $(\triangle N D>\triangle R D>\triangle T D)$ and it successively changed to $(\triangle R D>$ $\triangle N D>\triangle T D$ ), as is shown in Fig. 2.

Primary recrystallization texture was analyzed to evaluate the orientation distribution of Goss nuclei and that of coincidence $(\Sigma 9)$ grains. Figure 3(a) and (b) show the complete $\{100\}$ pole figures. Figure 4 shows the three dimensional orientation distribution of the center layer. It can be seen that the primary recrystallization texture of

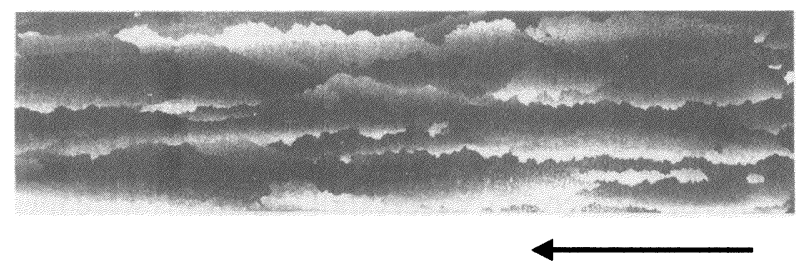

direction of temperature gradient

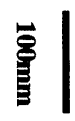

FIGURE 1 Macrostructure of secondary recrystallized grains in the temperature gradient annealing. 


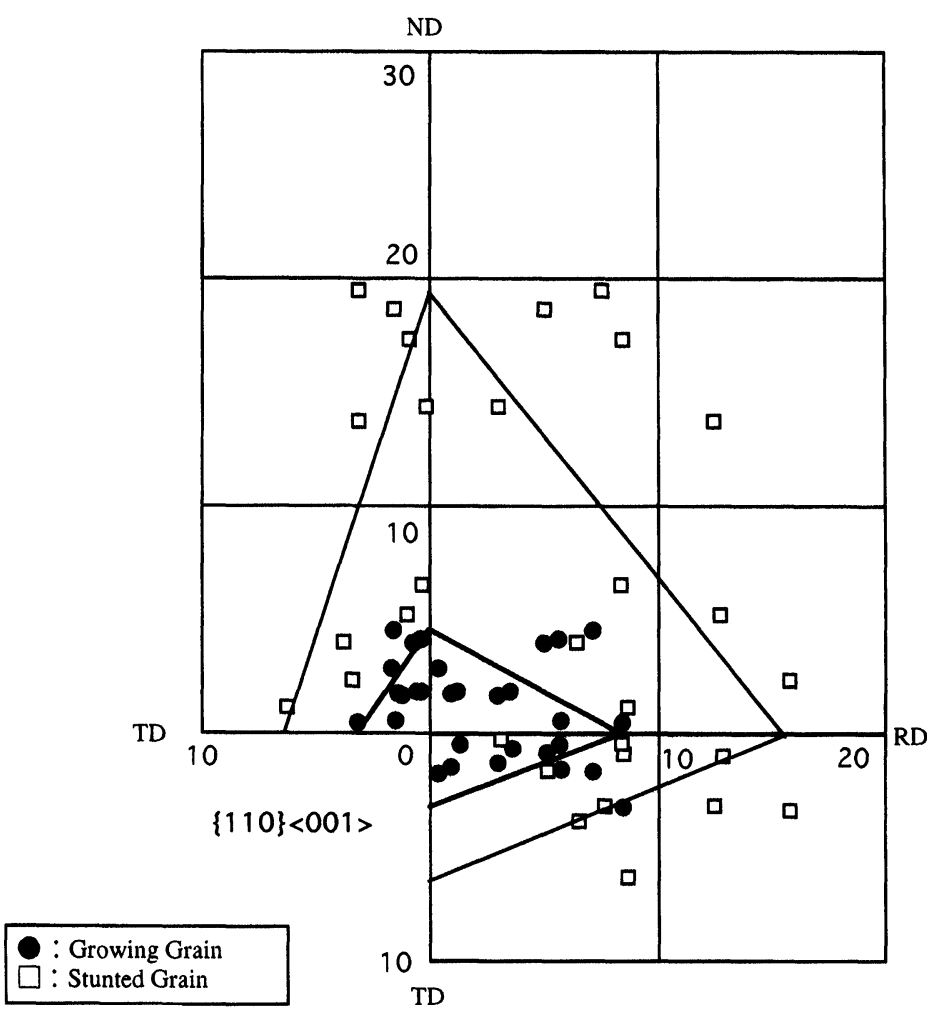

FIGURE 2 Change of orientation distribution of secondary recrystallized grains during grain growth.

the specimen consists of two major components; (a) $\gamma$-fiber with the peak of $\{111\}\langle 112\rangle$ and (b) rotated $\alpha$-fiber, which is rotated about $20^{\circ}$ around the ND axis, with the peak of $\{411\}\langle 148\rangle$. These two peaks, $\{111\}\langle 112\rangle$ and $\{411\}\langle 148\rangle$, are calculated to have a $\Sigma 9$ coincidence relationship to Goss orientation, and therefore they are important for the grain growth behavior. It has been reported that the Goss grains nucleate in the surface layer (Matsuo et al., 1982; Inokuti et al., 1982), hence, estimation of the orientation distribution of Goss nuclei was carried out by the pole figure of the surface layer and that of coincidence grains by the average pole figure of both layers. It can be seen from Fig. 5 that the orientation distribution of Goss nuclei and that of coincidence grains are quite different. The orientation distribution of Goss nuclei has a concave shape and that of coincidence grains 


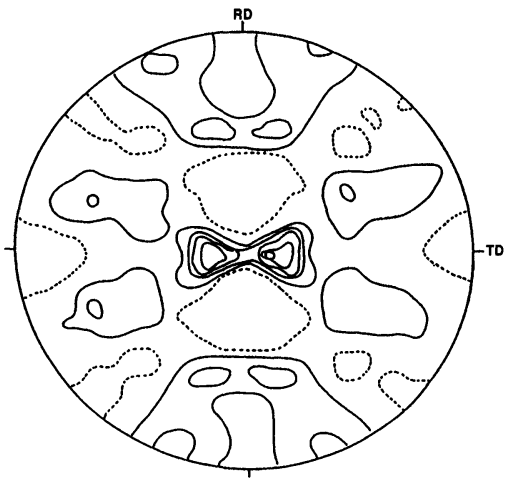

(a) surface layer

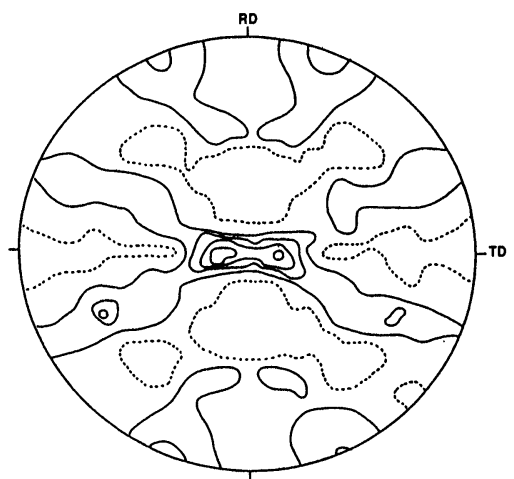

(b) center layer

FIGURE 3 Complete $\{100\}$ pole figures of primary recrystallization texture; (a) surface layer, (b) center layer.

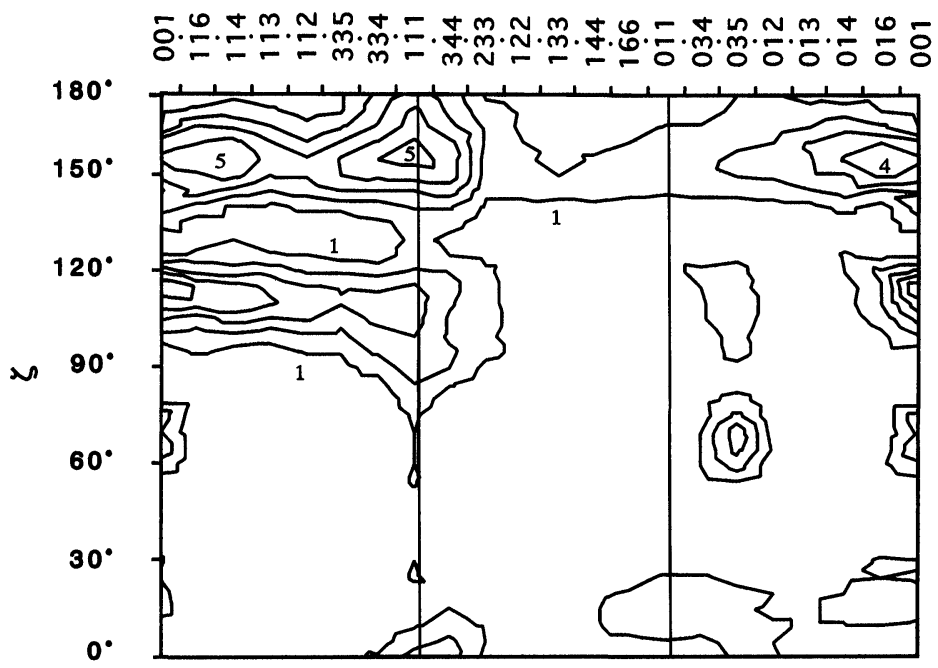

FIGURE 4 Three dimensional orientation distribution of primary recrystallization texture.

a convex shape and they are qualitatively estimated as $(\triangle \mathrm{ND}>$ $\triangle \mathrm{RD}>\triangle \mathrm{TD})$ and $(\triangle \mathrm{RD}>\triangle \mathrm{TD} \sim \triangle \mathrm{ND})$, respectively.

Thus, with the comparison between the orientation distribution of secondary recrystallized grains and those of Goss nuclei and coincidence grains in the primary recrystallized matrix, it is concluded that 


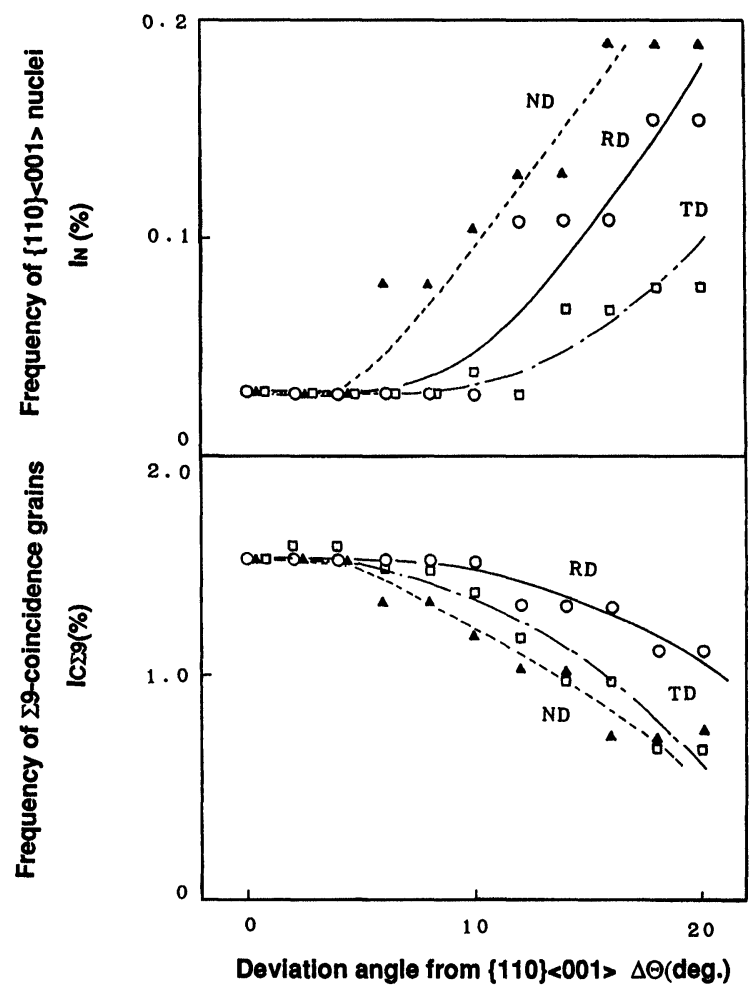

FIGURE 5 Orientation distribution of $\{110\}\langle 001\rangle$ nuclei and that of $\Sigma 9$ coincidence grains in the primary recrystallization texture.

the orientation distribution of secondary recrystallized grains corresponds to that of Goss nuclei at the initial stage of secondary recrystallization and it changes successively to that of matrix coincidence grains as grains grow.

\subsection{Orientation Selectivity Behavior During Grain Growth}

As shown in Fig. 1, sharper Goss grains which have higher frequencies of coincidence grains in the matrix were selected at the initial stage of grain growth among the several grains which were nucleated at the sheet edge, and after that they grew stationary to form the columnar structure. For the investigation of this selective growth mechanism, 
some specimens were quenched in the heating stage of the annealing. Figure 6(a) and (b) show the macrostructures of the growth front of the secondary recrystallized grains. It can be seen from these figures that secondary recrystallization temperature, which is defined as the growth front temperature, takes a specific value depending on the orientation of the secondary recrystallized grain. The secondary recrystallization temperatures of the secondary recrystallized grains in this system were in the range from $1050^{\circ} \mathrm{C}$ to $1065^{\circ} \mathrm{C}$. Secondary recrystallization temperatures of the sharper Goss grains are around $1050^{\circ} \mathrm{C}$, which are about $10^{\circ} \mathrm{C}$ lower than those of the deviated Goss grains. Depending
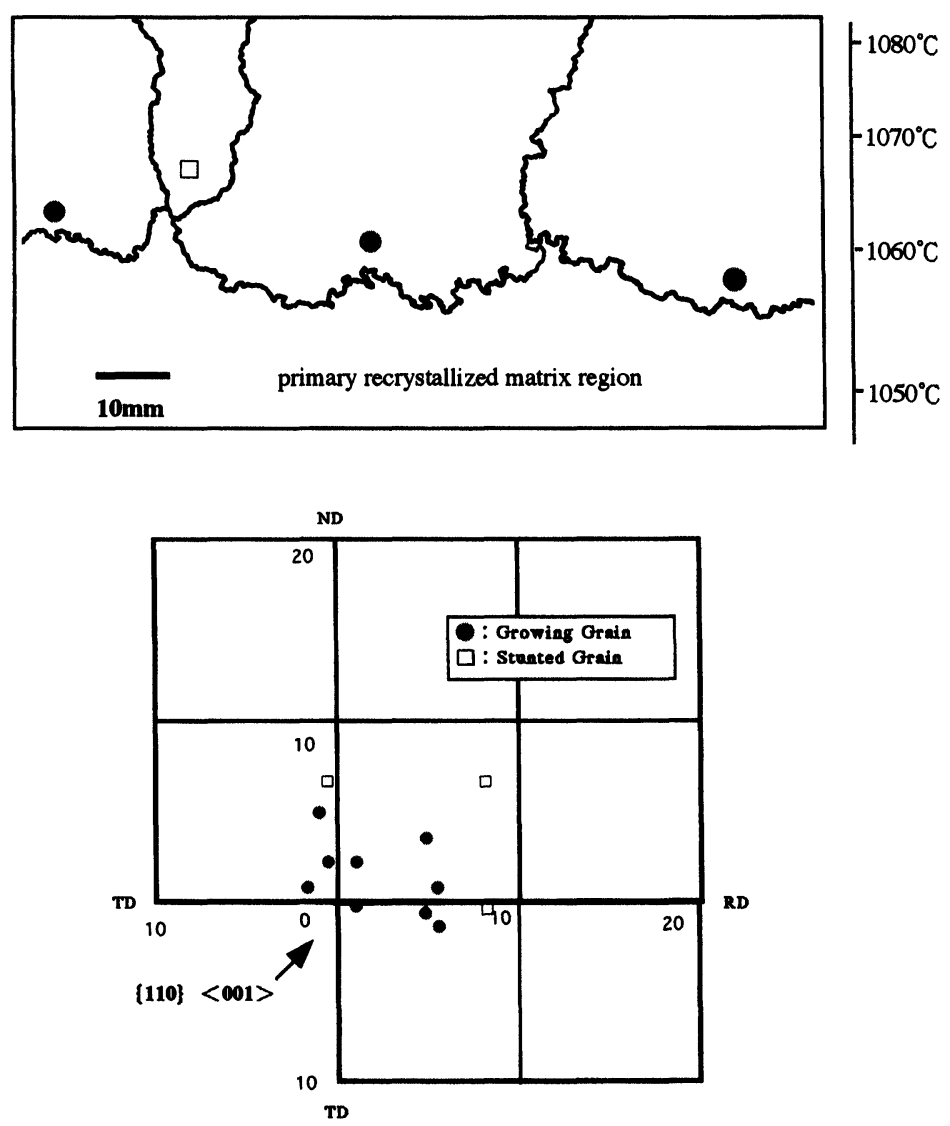

FIGURE 6(a) 

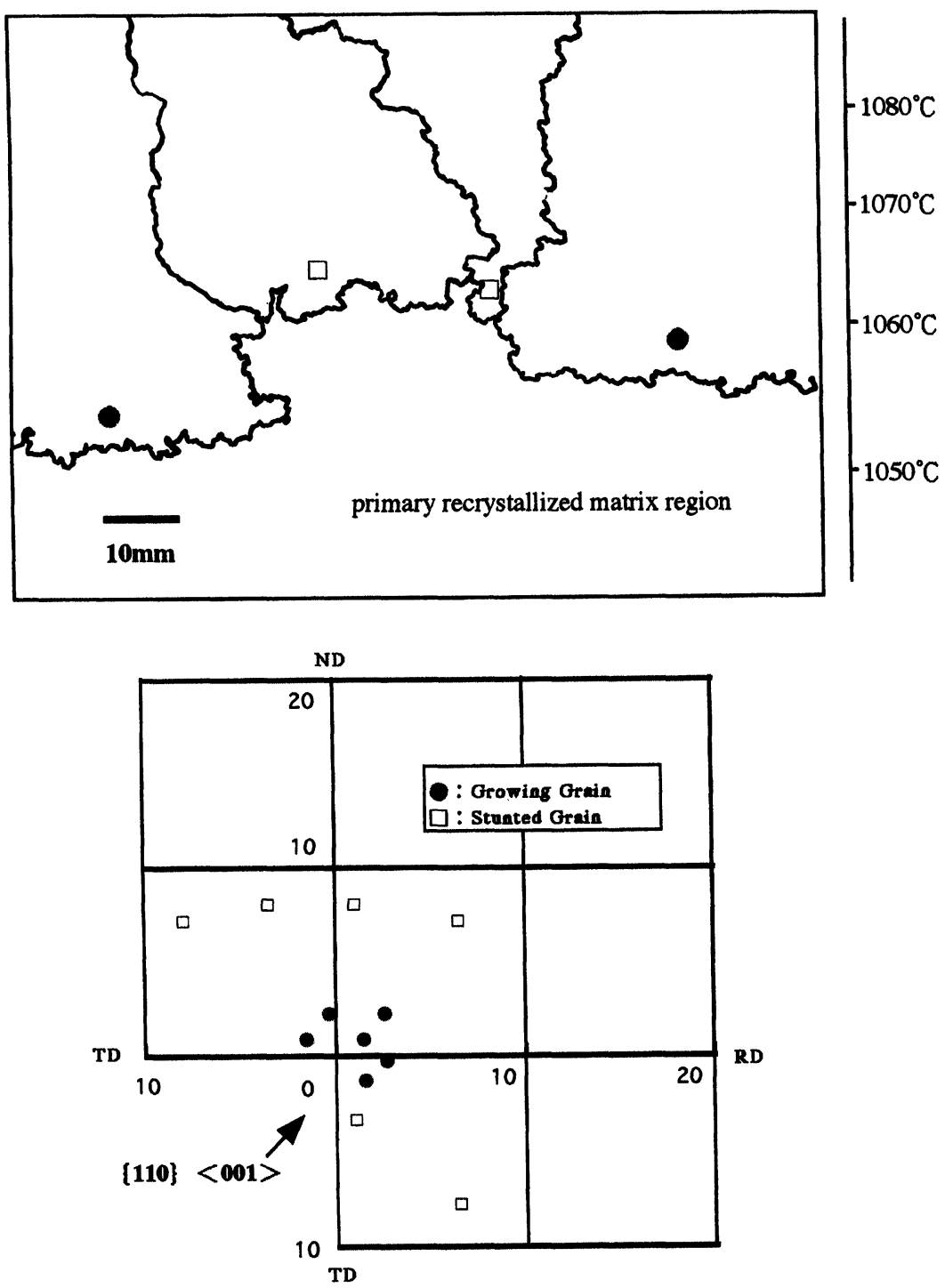

FIGURE 6(b)

FIGURE 6 Macrostructures of interface between the secondary recrystallized grains and primary recrystallized matrix and orientation distribution of secondary recrystallized grains. 
on the difference of secondary recrystallization temperature, sharper Goss grains grew preferentially by expanding the growth front and consuming the primary matrix region earlier than the deviated Goss grains. In the end, sharper Goss grains, which have the same secondary recrystallization temperature, grew stationary along the temperature gradient direction.

It is concluded that the secondary recrystallization temperature is the important parameter which dominates the selective growth behavior.

\subsection{Mechanism of Orientation Selectivity.}

Secondary recrystallization phenomena are described by the modified Hillert's model (Hillert, 1965; Abbruzzese and Lücke, 1986; Nakayama and Ushigami, 1986), which takes the textural effect into account. Grain growth rate of the secondary recrystallized grain is described by the following equations,

$$
\begin{gathered}
\mathrm{d} R / \mathrm{d} t=M P, \\
P=A E\left\{\left(1 / R_{\mathrm{c}}\right)-(K / R)-(K Z / A)\right\},
\end{gathered}
$$

where $P$ is the total driving force of secondary recrystallized grain, $R$ is the radius of secondary recrystallized grain, $R_{\mathrm{c}}$ is the critical radius of primary recrystallized matrix grains, $A$ is the geometrical factor, $Z$ is the Zener term, $M$ is the mobility, $E$ is the average grain boundary energy of primary recrystallized matrix, and $K$ is the ratio of secondary recrystallized grain boundary energy to that of matrix primary recrystallized grain boundary energy $(E)$.

As can be seen from Eq. (2) and Fig. 7, total driving force of secondary recrystallized grain consists of three components. The first $\left(P_{1}\right)$ is the driving force derived from the tension of grain boundaries of the primary recrystallized matrix. The second $\left(P_{2}\right)$ is the retarding force derived from the shrinking force of the secondary recrystallized grain itself. The third $\left(P_{3}\right)$ is the retarding force by the pinning effect of second phase particles.

It can be deduced from Eq. (2) that the driving force from the grain boundary tension of the matrix grains takes the same value irrespective of the orientation of the secondary recrystallization grains. Two modes of preferred grain growth are recognized as: (1) "oriented nucleation 


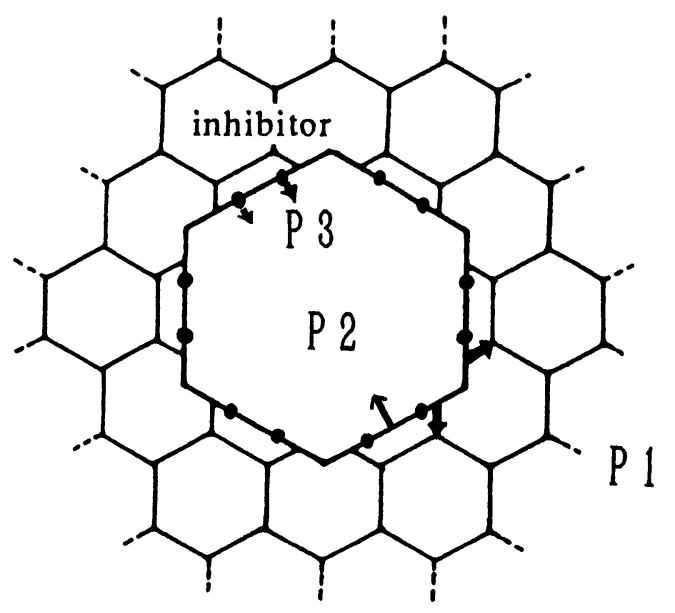

FIGURE 7 Schematic illustration of the forces which affect the secondary recrystallized grain.

derived from the size effect" (second term) and (2) "oriented growth derived from the inhibition effect" (third term).

In the "nucleation stage" of secondary recrystallization, where the grain size of the secondary recrystallized grain has the same order of magnitude compared with that of primary recrystallized matrix grains, the second component of the Eq. (2) takes an important role. Then, size and number of secondary recrystallization nuclei dominate the preferred grain growth behavior because this "size advantage effect" is inversely proportional to the grain size.

On the other hand, in the "grain growth stage", where the grain size of the secondary recrystallized grain becomes quite large compared with that of primary recrystallized matrix grains, the second term becomes negligible and the third component of Eq. (2) which represents the "inhibition effect" dominates the grain growth behavior. Then, the primary recrystallized grains which have coincidence relationship to secondary recrystallized grain takes an important role. It is reported that the coincidence boundary (or special boundary) has a lower grain boundary energy. Hence, the sharper Goss grains which have a higher frequency of coincidence grains to primary recrystallized matrix have the lower statistical grain boundary energy $(K E)$. Pinning effect derived from the second phase particles is estimated by the interaction energy 
between the grain boundary and precipitates, which is evaluated by the product of statistical grain boundary energy of the secondary recrystallized grain and area of cross section of second phase particles (Zener term). Thus, it is deduced that sharper Goss grains suffer lower pinning effect from second phase particles.

In the process of grain oriented silicon steel, one of the requisites for secondary recrystallization is the presence of fine precipitates to inhibit the grain growth of matrix grains (May and Turnbull, 1958). As is demonstrated in Fig. 8, nitrides such as $\mathrm{Al}(\mathrm{Si}) \mathrm{N}$ and $(\mathrm{Si}, \mathrm{Mn}) \mathrm{N}$ stagnate the grain boundary migration and act as inhibitors in this system. Figure 9 shows the change of nitrogen content of the specimen measured by chemical analysis. The inhibition effect decreases with increasing temperature. Secondary recrystallization temperatures of the secondary recrystallized grains are in the temperature range from $1050^{\circ} \mathrm{C}$ to $1065^{\circ} \mathrm{C}$, therefore it is concluded that secondary recrystallization proceeds during the deteriorating stage of inhibition effect. Secondary recrystallization temperatures of sharper Goss grains are lower than those of deviating Goss grains. This fact suggests that the sharp Goss grains start to grow in the stage of higher inhibition effect due to the grain boundary characteristics.

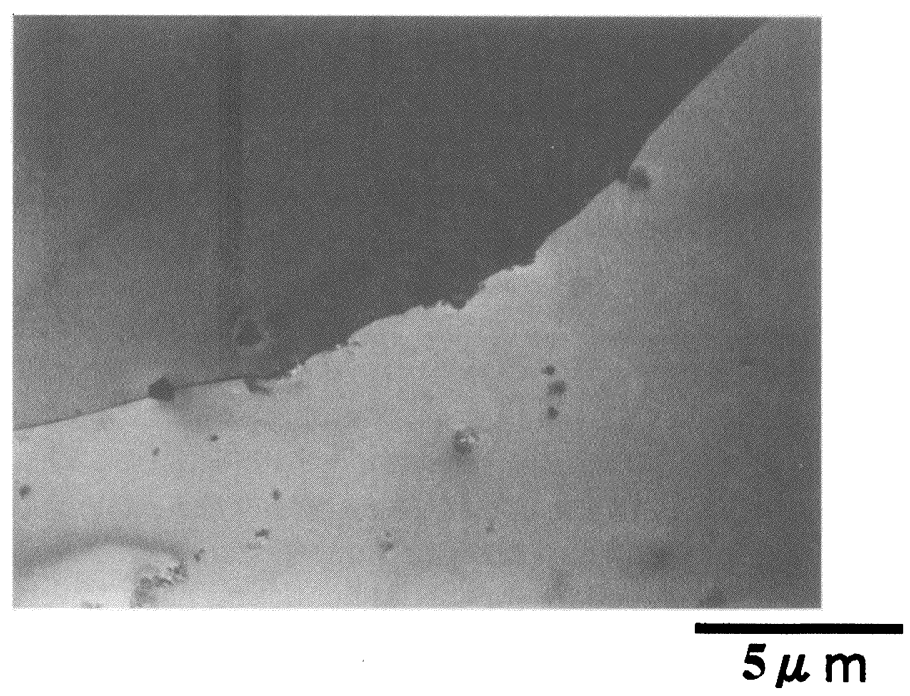

FIGURE 8 Transmission electron micrograph which shows the pinning effect of precipitates. 


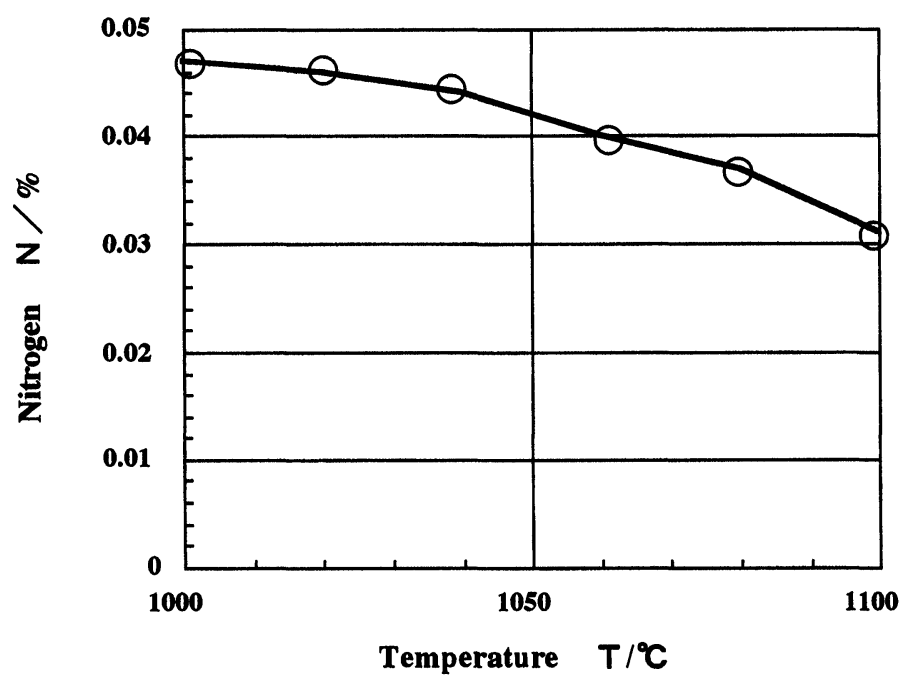

FIGURE 9 Change of nitrogen content during the seating stage of annealing.

As a summary, the mechanism of selective growth of the temperature gradient annealing is schematically illustrated in Fig. 10. Precise Goss grains, which have higher frequency of coincidence grains in the matrix, start to grow at lower temperatures and thus grow preferentially by expanding the growth front into the deviating Goss grains.

The present authors have proposed the same model of secondary recrystallization from the experimental results of in situ observation of secondary recrystallization by synchrotron X-ray topography (Ushigami et al., 1991; 1994). The $\{110\}\langle 001\rangle$ secondary recrystallized grain which has higher frequency of coincidence grains in primary recrystallized matrix starts to migrate at lower temperatures compared with the $\{110\}\langle 115\rangle$ grain by breaking away the inhibition effect from second phase particles.

When the temperature gradient annealing was applied to the CGO type process, the selective growth behavior which is described in this paper was not observed. This result suggests that sharp orientation distribution of coincidence grains in the primary recrystallized matrix which is the characteristic feature of the primary recrystallization texture in the HI-B type process is indispensable for the selective growth of Goss grains. 


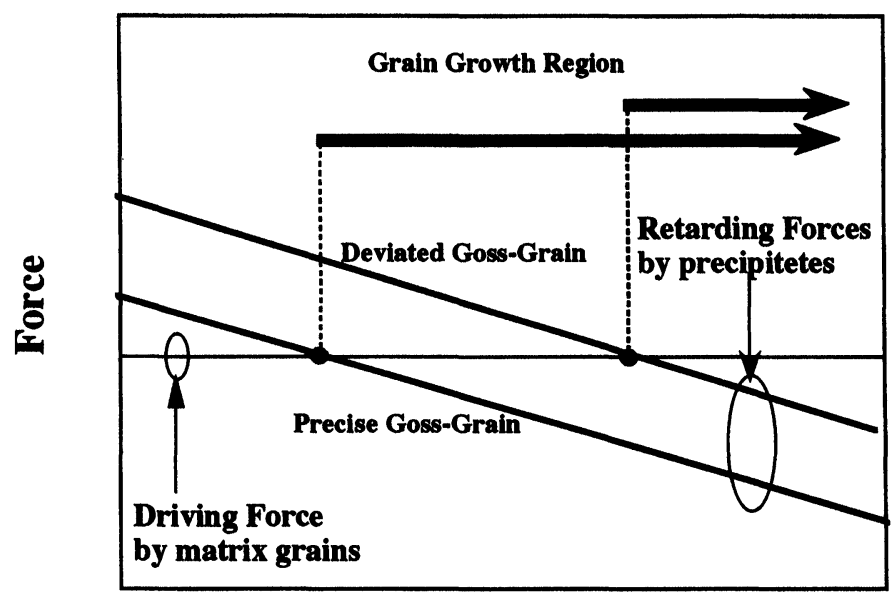

Temperature

FIGURE 10 Schematic diagram of orientation selectivity during grain growth of secondary recrystallization.

\section{CONCLUSIONS}

Selective growth behavior of Goss grains was investigated in the temperature gradient annealing.

(1) Goss grains selectively grow in the grain growth process; the average deviation angle becomes smaller and orientation distribution of secondary recrystallized grains changes corresponding to that of coincidence grains in the primary recrystallized matrix.

(2) Secondary recrystallization temperature is the dominant parameter for selective growth behavior. Sharper Goss grains grow at lower temperatures and expand their territories of grain growth.

(3) The orientation selectivity is explained by the modified Hillert's model of grain growth. Sharper Goss grains, which have higher frequency of coincidence grains in the primary matrix, suffer lower pinning effect from precipitates and thus grow preferentially at lower temperatures.

(4) Selective growth of sharper Goss grains takes a dominant role of texture control in the HI-B process, which is characterized by the 
sharp orientation distribution of coincidence grains in the matrix primary recrystallization texture.

\section{References}

Abbruzzese, G. and Lücke, K. (1986). Proc. 7th RISO Int. Symp. of Met. and Mat. Sci., p. 1.

Arai, S., Ushigami, Y. and Takahashi, N. (1996). Mat. Sci. Forum, 204-206, 617.

Flowers, J.W. and Heckler, A.J. (1976). IEEE Trans. on Mag. MAG-12, p. 846.

Goss, N.P. (1934). U.S.P. No. 1,965,559.

Goss, N.P. (1935). Trans. ASM, 23, 511.

Harase, J., Shimizu, R. and Watanabe, T. (1986). Proc. 7th RISO Intern. Symp. on Met. Mater. Sci., p. 343.

Hillert, M. (1965). Acta Met., 13, 227.

Inokuti, Y., Maeda, C., Itoh, Y. and Shimanaka, H. (1982). Proc. 6th Intern. Conf. on Textures of Materials, p. 948.

Littmann, M.F. (1967). J. Appl. Phys., 38, 1104.

Matsuo, M., Sakai, T., Tanino, M., Shindo, T. and Hayami, S. (1982). Proc. 6th Intern. Conf. on Textures of Materials, p. 918.

Matsuo, M. (1989). ISIJ International, 29, 809.

May, J.E. and Turnbull, D. (1958). Trans. Met. Soc. AIME, 212, 769.

Mishra, S., Därmann, C. and Lücke, K. (1984). Acta Met., 32, 2185.

Mishra, S., Därmann, C. and Lücke, K. (1986). Met. Trans., 17A, 1301.

Nakayama, T. and Ushigami, Y. (1986). Proc. 7th RISO Intern. Symp. on Met. and Mat. Sci., p. 463.

Nozawa, T., Nakayama, T., Ushigami, Y. and Yamamoto, T. (1986). J. Mag. Mag. Mat., 58, 67.

Rouag, N. and Penelle, R. (1989). Textures and Microstructures, 11, 203.

Ruer, D. and Baro, R. (1977). J. Appl. Crystallogr., 10, 458.

Shinozaki, M., Matoba, I., Kan, T. and Gotoh, T. (1978). Trans. JIM, 19, 85.

Taguchi, S. and Sakakura, A. (1969). J. Appl. Phys., 40, 1539.

Ushigami, Y., Suga, Y., Takahashi, N., Kawasaki, K., Chikaura, Y. and Kii, H. (1991). J. Mater. Eng., 13, 113.

Ushigami, Y., Kawakaki, K., Nakayama, T., Suga, Y., Harase, J. and Takahashi, N. (1994). Mat. Sci. Forum, 157-162, 1081.

Ushigami, Y., Kurosawa, F., Masui, H., Suga, Y. and Takahashi, N. (1996). Mat. Sci. Forum, 204-206, 593.

Yamamoto, T., Taguchi, S., Sakakura, A. and Nozawa, T. (1972). IEEE Trans. on Mag., MAG-8, p. 677. 